\title{
PROSPECTS FOR APPLYING LASER-MECHANICAL METHODS TO THE IMPROVEMENT OF THE PHYSICAL PROPERTIES OF TRANSFORMER STEEL
}

\author{
Yu. N. Dragoshanskii, V. I. Pudov, Yu. Ya. Reutov, A. S. Doroshek \\ M.N. Miheev Institute of Metal Physics, Ural Branch of the Russian Academy of Sciences, \\ 18 S. Kovalevskoy St., 620990, Ekaterinburg, Russian Federation \\ *Corresponding author. E-mail: drago@imp.uran.ru,pudov@imp.uran.ru \\ Address for correspondence: 18, S. Kovalevskoy st., 620990, Ekaterinburg, Russian Federation \\ Tel: +7 (343) 3783694
}

\begin{abstract}
The influence of the (110) [001] edge crystallographic textures on the prospects of reducing magnetic losses in local laser processing, mechanical scribing and local bending, solving the problem of improving the functional characteristics of $\mathrm{Fe}-3 \% \mathrm{Si}$ anisotropic magnets and amorphous alloys, is investigated. It is shown that the complex effect of laser treatment and subsequent thermo magnetic treatment in an alternating magnetic field makes it possible to reduce the magnetic losses of $P_{1.7 / 50}$ in steels of higher grades with an edge texture close to the optimal one (grade 3409, $\mathrm{B}_{800}=1.93 \mathrm{~T}$ ) by $14-18 \%$ and $18-26 \%$ in a thin amorphous ribbon of the $\mathrm{Fe}_{81} \mathrm{Si}_{7} \mathrm{~B}_{12}$ alloy.
\end{abstract}

Keywords: electric steels, structure, domains, laser-mechanical treatment, physical properties.

DOI: $10.17804 / 2410-9908.2017 .6 .048-056$

\section{References}

1. Starodubtsev Yu.N. Magnitomyagkie Materialy [Soft Magnetic Materials: Encyclopaedic hand-book]. Moscow, Tekhnosfera Publ., 2011, 659 p. (In Russian).

2. Ichiyama T. Structure and control of magnetic domain in grain-oriented silicon steel. Journal of the Iron and Steel Institute of Japan, 1983, vol. 69, iss. 8, pp. 895-902.

3. Stal elektrotekhnicheskaya kholodnokatanaya anizotropnaya tonkolistovaya. GOST 21427.1-83 [Cold-rolled anisotropic electrical-sheet steel. Specifications]. (In Russian).

4. Pudov V.I., Dragoshanskii Yu.N. Promising applications of amorphous crystalline coatings for soft magnetic alloys. Uprochnyayushchie Tekhnologii i Pokrytiya, 2013, iss. 8, pp. 44-48. (In Russian).

5. Dragoshanskii Yu.N., Pudov V.I. Improving the magnetic structure and properties of soft magnetic alloys by modification of their surface. Fizika i Khimiya Obrabotki Materialov, 2013, no. 3, pp. 48-52. (In Russian).

6. Dragoshanskii Yu.N., Pudov V.I., Karenina L.S. Electrical steel domain optimization and magnetic loss reduction by active coating and laser treatment. Izvestiya RAN. Seriya Fizicheskaya, 2013, vol. 77, iss. 10, pp. 1286-1288. (In Russian).

7. Dragoshanskii Yu.N., Pudov V.I. Physical basis for improving the electromagnetic properties of soft magnetic materials. In: Modern methods and technologies for the creation and processing of materials: IX International Scientific and Technical Conference, Minsk, September 19-22, 2014, Minsk, FTI NAN Belarusi Publ., 2014, pp. 137-144. (In Russian).

8. Pudov V.I., Dragoshanskii Yu.N. Method of manufacturing anisotropic electrical steel. RF Patent 2569260. (In Russian).

9. Dragoshanskii Yu.N., Pudov V.I. Formation of arranged defects and improvement of the magnetic properties of soft magnetic materials with different magnetic texture. In: Modern Methods and Technologies for the Creation and Processing of Materials: IX International Scientific and Technical Conference, Minsk, September 14-16, 2016, FTI NAN Belarusi Publ., 2016, pp. 68-73. (In Russian).

Dragoshanskii Yu.N. et al. / Prospects for applying laser-mechanical methods to the improvement of the physical properties of transformer steel 
http://dream-journal.or"]

10. Dragoshanskii Yu.N. Optimization of the domain structure and reduction of magnetic losses in anisotropic electric steels and alloys. In: Magnetizm Perekhodnykh Metallov $i$ Splavov [Magnetism of Transition Metals and Alloys, ed. Yu.A. Izyumov]. Ekaterinburg, UB RAS Publ., 2000, pp. 146-157. (In Russian).

11. Dragoshanskii Yu.N., Pudov V.I. Influence of laser processing and magnetically active inorganic coatings on the dynamic magnetic properties of soft-magnetic materials. Inorganic Materials, 2013, vol. 49, iss. 7, pp. 668-675. DOI: 10.1134/S0020168513070029 
Подана в журнал: 30.11 .2017

УДК 621.758;537.622

DOI: $10.17804 / 2410-9908.2017 .6 .048-056$

\title{
ПЕРСПЕКТИВЫ ПРИМЕНЕНИЯ ЛАЗЕРНО-МЕХАНИЧЕСКИХ МЕТОДОВ ДЛЯ УЛУЧШЕНИЯ ФИЗИЧЕСКИХ СВОЙСТВ ТРАНСФОРМАТОРНОЙ СТАЛИ
}

\author{
Ю. Н. Драгошанский, В. И. Пудов, Ю. Я. Реутов, А. С. Дорошек \\ Институт физики металлов им. М.Н. Михеева Уральского отделения Российской академии наук, \\ Екатеринбург, Российская Федерачия

\begin{abstract}
*Ответственный автор. Электронная почта: drago@imp.uran.ru, pudov@imp.uran.ru Адрес для переписки: ул. С. Ковалевской, 18, 620990, Екатеринбург, Российская Федерация
\end{abstract} \\ Тел.: +7 (343) 378-36-94
}

Исследовано влияние ребровой кристаллографической текстуры (110)[001] на перспективы снижения магнитных потерь при локальной лазерной обработке, механическом скрайбировании и локальных изгибах, решающие проблему улучшения функциональных характеристик анизотропных магнетиков $\mathrm{Fe}-3 \% \mathrm{Si}$ и аморфных сплавов. Показано, что комплексное воздействие лазерной обработки и последующей термомагнитной обработки в переменном магнитном поле позволяет в сталях высших марок с ребровой текстурой, близкой к оптимальной (марка $3409, \mathrm{~B}_{800}=1,93$ Тл), снизить магнитные потери $P_{1,7 / 50}$ на $14-18$ \% и на $18-26 \%$ в тонкой аморфной ленте сплава $\mathrm{Fe}_{81} \mathrm{Si}_{7} \mathrm{~B}_{12}$.

Ключевые слова: электротехнические стали, структура, домены, лазерно-механические воздействия, физические свойства.

\section{1. Введение}

В настоящее время в промышленном производстве холоднокатаной анизотропной электротехнической стали (АЭТС - сплав $\mathrm{Fe}-3 \% \mathrm{Si}$ ) для получения высокой магнитной проницаемости вдоль направления прокатки используют, в основном, один из двух технологических способов - с двукратной прокаткой и промежуточным отжигом или с однократной силовой прокаткой [1]. Кристаллическая структура и магнитные свойства сталей этих двух технологий производства имеют существенные различия. Второй способ обеспечивает получение высокопроницаемой анизотропной стали с совершенной кристаллографической текстурой типа «Хай-Би» [2], при которой индукция в магнитном поле $800 \mathrm{~A} / \mathrm{m} \mathrm{B}_{800}=1,92-1,98$ Тл и выше, по сравнению с аналогичной величиной 1,82-1,88 Тл, достигаемой при первом способе [3].

При этом электротехнические стали имеют крупное зерно с острой ребровой текстурой (110) [001], осью легкого намагничивания [001], ориентированной вдоль направления прокатки, и крупными полосовыми $180^{\circ}$ доменами. В результате при эксплуатации стали возрастают скорости перемещения границ доменов, что приводит к увеличению магнитных потерь.

Удельные магнитные потери $P_{1,7 / 50}$ (при индукции 1,7 Тл и частоте перемагничивания 50 Гц) и магнитная индукция в слабых $B_{100}$ или в средних $B_{800}$ полях (при напряженности $100 \mathrm{~A} / \mathrm{м}$ и $800 \mathrm{~A} / \mathrm{M})$ являются основными характеристиками качества стали. Магнитные потери $P$ разделяют на гистерезисные и вихретоковые, причем основная доля приходится на вихретоковые.

Величина вихретоковых магнитных потерь $\delta \sim E^{2} / \rho$, где $E$ - напряженность электрического поля; $\rho$ - удельное сопротивление. Однако $E \sim d \Phi / d t \sim v$, где $v$ - скорость перемеще-

Dragoshanskii Yu.N. et al. / Prospects for applying laser-mechanical methods to the improvement of the physical properties of transformer steel 
ния доменной стенки. Учитывая, что $v \sim f / N \sim D f$, где $N-$ количество доменов на единице ширины образца; $f$ - частота перемагничивания для магнитных потерь, то можно записать:

$$
\delta \sim D^{2} f^{2} / \rho .
$$

Таким образом, уменьшение ширины основных полосовых $180^{\circ}$ доменов приводит к снижению магнитных потерь.

В связи с этим, для лент высоко текстурованных АЭТС, имеющих слабые магнитные поля рассеяния на поверхности и крупные магнитные полосовые домены (шириной D 1 мм), активно разрабатываются способы уменьшения размеров доменов и вихретоковой составляющей магнитных потерь за счёт формирования протяженных упорядоченных зон, структура которых отлична от строения окружающей матрицы.

К таким способам, активно изучаемым в настоящее время, относятся деформационнотекстурующие воздействия, например, локальная лазерная обработка (ЛЛО), механическое скрайбирование [4-8], локальные или волнообразные изгибы лент стали [9]. При использовании каждого из них в лентах анизотропной стали формируют зоны магнитных полей рассеяния, локальные из которых ориентированы поперёк оси текстуры на расстояниях, меньших размеров зерна. В процессе перемагничивания лент под воздействием этих полей возникает и вырастает масса зародышей перемагничивания, образуя измельчённые полосовые $180^{\circ}$ домены. Движение их границ происходит с уменьшенными скоростями, что и приводит к снижению вихретоковых и полных магнитных потерь энергии.

Цель работы - исследования доменной структуры и магнитных потерь монокристальных, поликристаллических лент сплава $\mathrm{Fe}-3 \% \mathrm{Si}$ и аморфного сплава $\mathrm{Fe}_{81} \mathrm{Si}_{7} \mathrm{~B}_{12}$ в результате воздействия механического скрайбирования, ЛЛО, термомагнитой обработки (ТМО) и растяжения.

\section{2. Материалы и методы исследования}

Исследование проводили на образцах в виде монокристальных полос стали размерами $120 \times 10 \times 0,2$ мм, поверхность (110) которых составляла с направлением оси [001] углы $\beta=0$; $1 ; 2 ; 3 ; 4 ; 5 ; 6^{\circ}$ [9], на высоко текстурованных поликристаллических лентах стали марки 3409 толщиной 0,27 мм, с размерами зёрен 20-30 мм и на лентах аморфного сплава $\mathrm{Fe}_{81} \mathrm{Si}_{7} \mathrm{~B}_{12}$ толщиной 20-22 мкм, шириной 5-10мм. Магнитные потери $P_{1,7 / 50}$ в стали и $P_{1 / 400}$ в аморфном сплаве измеряли с помощью магнитоизмерительной установки МК-4Э. Магнитную доменную структуру выявляли магнитопорошковым и магнитооптическим методами.

\section{3. Результаты и их обсуждение}

Выявлено, что в лентах АЭТС снижение степени совершенства ребровой кристаллографической текстуры, в частности, увеличение $\beta^{\circ}$ (угла отклонения оси [001] и намагниченности зёрен от поверхности ленты) приводит к появлению магнитных полей рассеяния над поверхностью ленты. При этом постепенно сужаются основные полосовые домены 0,8-0,2 мм, что ведёт к уменьшению скоростей движения их границ и вихретоковой составляющей магнитных потерь при перемагничивании (рис. 1).

Одновременно с этим на поверхности появляются замыкающие каплевидные домены и растёт их плотность 9-40 \% площади, что вызывает задержки и скачки смещающихся доменных границ, увеличивая гистерезисную составляющую магнитных потерь. В результате наблюдается нелинейная зависимость магнитных потерь от величины угла $\beta$ с минимумом при оптимальной величине $\beta=2^{\circ}$, составляющем 0,65 Вт/кг в монокристальных образцах стали, что на $42 \%$ меньше, чем в образцах идеальной ребровой ориентации, где $\beta=0^{\circ}, P_{1,7 / 50}=1,18 \mathrm{Bт} /$ кг (рис. 1 ).

Dragoshanskii Yu.N. et al. / Prospects for applying laser-mechanical methods to the improvement of the physical properties of transformer steel 


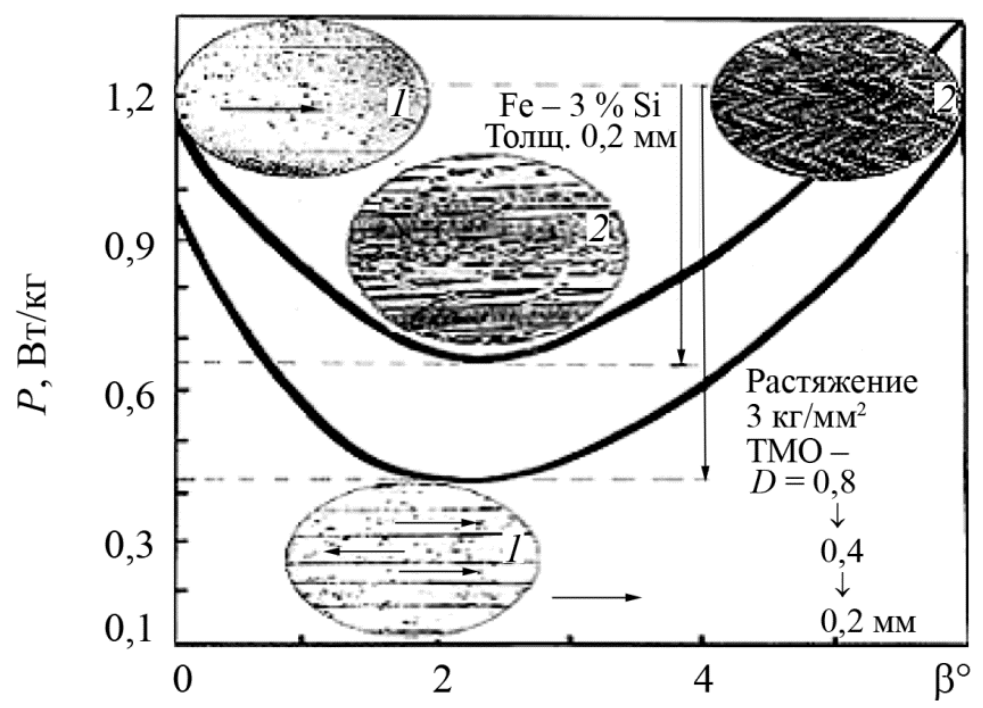

Рис. 1. Домены и магнитные потери монокристаллов $\mathrm{Fe}-3 \% \mathrm{Si}$ :

1 - полосовые $180^{\circ}$ домены с разной шириной и разной плотностью каплевидных замыкающих $180-90^{\circ}$ доменов (2) при увеличении угла $\beta: 0-6^{\circ}$

Упругое растяжение исследуемых монокристаллов $\sigma=20$ МПа вдоль оси текстуры приводит к уменьшению объёма каплевидных замыкающих доменов, связанных с внутри объёмными $90^{\circ}$ доменами поперечной намагниченности [10]. Возникающий при этом рост магнитостатической энергии образца компенсируется сужением основных полосовых доменов в 2-3 раза. Следовательно, уменьшается и величина магнитных потерь при перемагничивании (на 14-35 \%) с максимальным эффектом в образцах с оптимальной кристаллографической текстурой $\left(\beta=2^{\circ}\right)$, соответствующей минимуму магнитных потерь.

Механическое скрайбирование - нанесение резцом канавок глубиной 0,05 мм и шириной 0,3 мм поперек оси текстуры с промежутками 5 мм (рис. 2) также приводит к существенному уменьшению ширины полосовых доменов (в 1,5-2,5 раза) с максимумом эффекта $25 \%$ в образцах с идеальной ребровой ориентацией поверхности, имеющих наибольшую ширину исходных полосовых доменов. При этом вблизи зон деформации возникающие напряжения нарушают одноосную текстуру, искажают структуру магнитных доменов и увеличивают гистерезисную составляющую магнитных потерь. Низкотемпературный отжиг лент стали после скрайбирования позволяет уменьшить объём зон деформации и на 2-4 \% дополнительно снизить магнитные потери в материале.

Комплексное воздействие растяжения и скрайбирования образцов не является аддитивным, но обеспечивает более значительное снижение магнитных потерь: от $15 \%$ в образцах с максимальным отклонением намагниченности $\left(\beta=6^{\circ}\right)$ до $37 \%$ в образцах оптимальной ориентации $\left(\beta=2^{\circ}\right)$.

Эффект первого из воздействий существенно снижает эффект последующего. Отметим, что в заводских условиях растяжение тонколистовой стали обеспечивается нанесением магнитоактивного (растягивающего металл) электроизоляционного покрытия с коэффициентом теплового расширения (КТР), меньшим, чем КТР стали $\left(13 \times 10^{-6}\right)$ [11].

В поликристаллах стали основного состава $\mathrm{Fe}-3 \% \mathrm{Si}$ с достаточно крупным зерном 20-30 мм эффективным способом формирования деформированных зон оказывается бесконтактное ЛЛО. При ЛЛО наносимые на поверхность ленты стали поперечные (относительно оси прокатки) узкие зоны тепловой деформации с промежутками 5 мм, создают магнитные поля рассеяния и замыкания магнитного потока в этих участках лент, что в 2-4 раза уменьшает ширину полосовых $180^{\circ}$ магнитных доменов и, соответственно, мощность магDragoshanskii Yu.N. et al. / Prospects for applying laser-mechanical methods to the improvement of the physical properties of transformer steel 
нитных потерь. Продольное растяжение материала, возникающее между зонами сжатия лазерного воздействия, также способствует дроблению полосовых доменов.

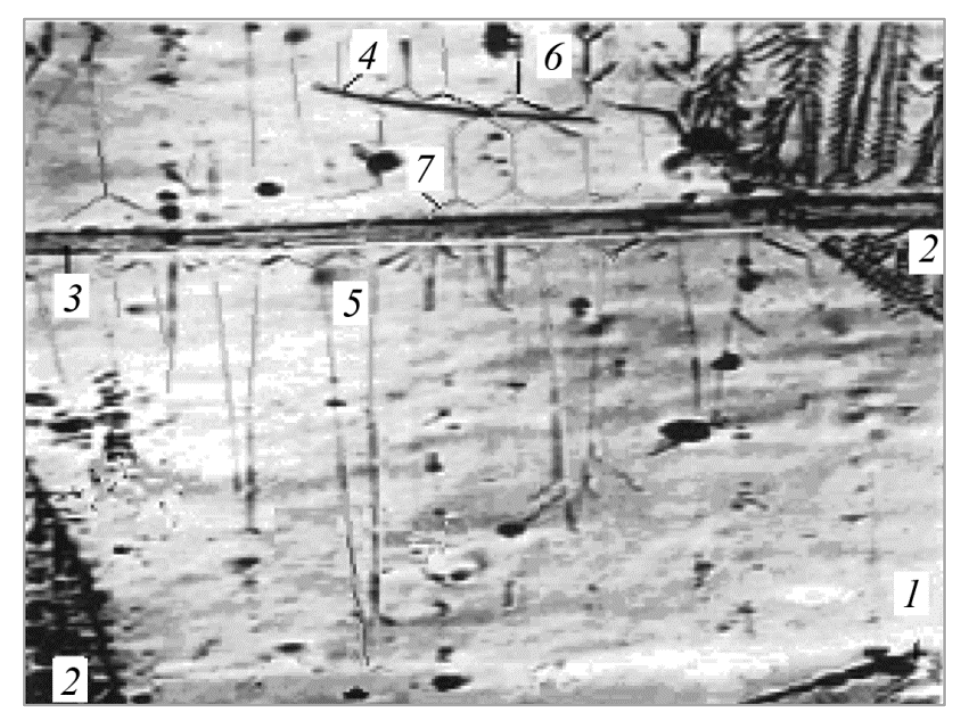

Рис. 2. Домены в поликристалле Fe-3 \% Si: 1 - зерно идеальной ориентации (110); 2 - зерна с разориентацией в листе на угол $\beta: 1-6^{\circ} ; 3$ - зона скрайбирования; 4 - поперечный линейный дефект, 5 - клиновидные $180^{\circ}$ домены; 6 - основные полосовые $180^{\circ}$ домены;

7 - призматические $90^{\circ}$ замыкающие домены $\times 30$

Эффект снижения магнитных потерь при использовании этого способа измельчения доменов, как и в случае монокристаллов [9], зависит от степени совершенства кристаллографической текстуры. Определением степени текстурованности стали может служить величина её индукции в магнитном поле напряженностью 800 A/м $\left(\mathrm{B}_{800}\right.$, Тл). Так, в лентах стали марки $3407\left(\mathrm{~B}_{800}=1,86\right.$ Тл), при толщине 0,27 мм и стандартном электроизоляционном покрытии (с растягивающим напряжением $\sigma \sim 6 \mathrm{MПа),} \mathrm{применение} \mathrm{ЛЛО} \mathrm{снижает} \mathrm{магнитные} \mathrm{потери} \mathrm{на}$ $8 \%$, а в стали с большей степенью текстурованности - марки 3409 ( $\mathrm{B}_{800}=1,93$ Тл) на $14 \%$ (табл.).

Таблица - Снижение магнитных потерь в сталях марок 3407, 3409 и в аморфной ленте $\mathrm{Fe}_{81} \mathrm{Si}_{7} \mathrm{~B}_{12}$ после ЛЛО и скрайбирования

\begin{tabular}{|c|c|c|c|c|c|c|}
\hline \multirow[t]{2}{*}{ Покрытие } & \multirow[t]{2}{*}{ Марка } & \multirow[t]{2}{*}{ Свойства } & \multicolumn{4}{|c|}{$\begin{array}{c}\text { Магнитные потери, Вт/кг } \\
\text { и их изменение (\%) при состояниях }\end{array}$} \\
\hline & & & Исх. & Скрайб. & ЛЛО & ЛЛО + ТМО \\
\hline \multirow{6}{*}{$\begin{array}{l}\text { Обычно- } \\
\text { eMg-P-B }\end{array}$} & \multirow{3}{*}{3407} & Индукция $\mathrm{B}_{800}$, Тл & 1,86 & 1,82 & 1,80 & 1,82 \\
\hline & & $P_{1,7 / 50,} \mathrm{BT} / \mathrm{\kappa} \Gamma$ & 1,18 & 1,12 & 1,09 & 1,04 \\
\hline & & $\Delta P, \%$ & - & 5 & 8 & 12 \\
\hline & \multirow{3}{*}{3409} & Индукция $\mathrm{B}_{800}$, Тл & 1,93 & 1,91 & 1,92 & 1,93 \\
\hline & & $P_{1,7 / 50,} \mathrm{BT} /$ кг & 1,06 & 0,98 & 0,1 & 0,87 \\
\hline & & $\Delta P, \%$ & - & 8 & 14 & 18 \\
\hline \multirow{2}{*}{\multicolumn{2}{|c|}{$\begin{array}{l}\text { Аморфная лента } \\
\text { Отжиг } 410^{\circ}-1 \text { ч }\end{array}$}} & $P_{1 / 400,}$ Вт/кГ & 2,08 & 1,81 & 1,71 & 1,54 \\
\hline & & $\Delta P, \%$ & - & 13 & 18 & 26 \\
\hline
\end{tabular}

Толщина 0,27 мм

Dragoshanskii Yu.N. et al. / Prospects for applying laser-mechanical methods to the improvement of the physical properties of transformer steel 
Последующая термомагнитная обработка - отжиг лент стали в переменном магнитном поле (напряжённость поля 1,4 кА/м, температура $400{ }^{\circ} \mathrm{C}$ ), - уменьшающая неоднородные внутренние напряжения и обеспечивающая дестабилизацию доменов, увеличивает эффекты снижения магнитных потерь на 4-6\%.

В ленте из аморфного сплава $\mathrm{Fe}_{81} \mathrm{Si}_{7} \mathrm{~B}_{12}$ создание поперечно ориентированных зон со структурой, изменённой лазерным воздействием или скрайбированием, также существенно снижает магнитные потери. При глубине воздействия 7-9 мкм и межзонными промежутками 3-5 мм снижение магнитных потерь, например $P_{1 / 400}$ составляет $12-16 \%$. Применение уже комбинированного воздействия в условиях создания поперечных зон при ЛЛО и отжиге в высокочастотном магнитном поле напряженностью 0,8 кА/м и частотой 50-60 кГц позволяет в ленте сплава снизить магнитные потери $\mathrm{P}_{1 / 400}$ практически на четверть (от 2, 08 до 1,54 Вт/кг) коэрцитивную силу $H_{\mathrm{c}}$ - в 4-5 раз и повысить начальную и максимальную магнитные проницаемости в 3-4 раза ( $\mu_{\max }$ от 96000 до 265000 отн. ед.). Однако при изготовлении магнитопровода из такой ленты, магнитные свойства существенно ухудшаются из-за появления напряжений в ленте от её изгиба. Поэтому требуется улучшать магнитные свойства и в изделиях.

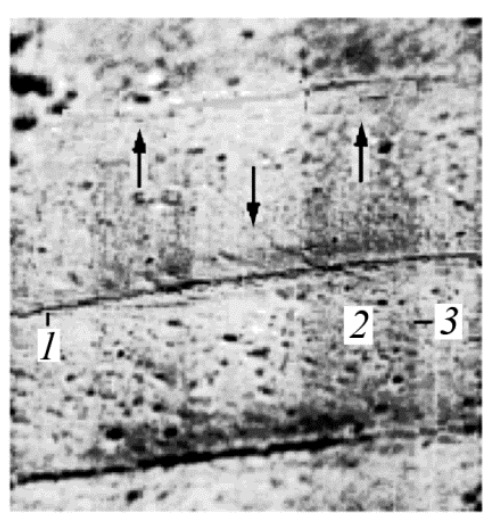

$a$

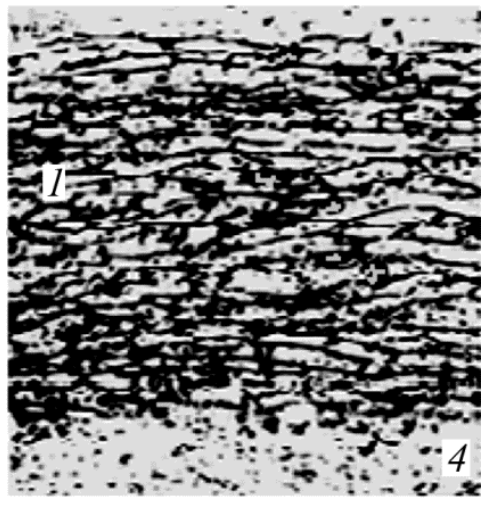

$\sigma$

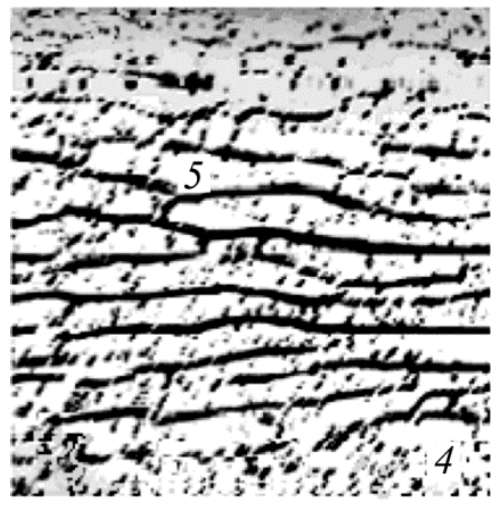

B

Рис. 3. Доменная и кристаллическая структура ленты стали при изгибной деформации: $a$ - зоны изгибной деформации до отжига (1), $180^{\circ}$ домены (2), границы доменов (3); $\sigma$ - ячеистая структура дислокаций зоны изгиба (1), межзонные промежутки (4) до отжига; в - дислокационная структура зоны изгиба (5), межзонные промежутки (4) после отжига. $\uparrow$ - направление намагниченности

Зоны механической деформации можно создавать локальными изгибами ленты [10], продвигаемой между валками, один из которых имеет зубчатую поверхность, а другой гуммирован (рис. 3). Однако и после низкотемпературного отжига (переход из напряжённого в менее напряжённое состояние) лента полностью не возвращается к плоскому состоянию, сохраняя остаточный изгиб кристаллической решётки, а полигональная структура деформированных зон, плавно переходящая к исходной структуре кристалла (рис. 3 б), не создаёт значительного рассеяния магнитного потока. В межзонных промежутках практически не образуются дополнительные зародыши перемагничивания, и снижение полных магнитных потерь составляет лишь 4-5 \%. Обнаружено, что механическое выпрямление рулонной кривизны отожжённой ленты в магнитопроводе (переход из не напряжённого в напряжённое состояние), наоборот, приводит к росту магнитных потерь и требует отжига.

Волнистая лента стали, подвергнутая до рекристаллизации плавным изгибам, создающим периодические поля рассеяния над лентой и сужение доменов, при её последующем механическом выпрямлении также требует отжига.

Dragoshanskii Yu.N. et al. / Prospects for applying laser-mechanical methods to the improvement of the physical properties of transformer steel 


\section{4. Выводы}

Таким образом, для уменьшения ширины полосовых магнитных доменов и магнитных потерь в анизотропных электротехнических сталях основного состава $\mathrm{Fe}-3$ \% $\mathrm{Si}$ способ локальной лазерной обработки оказывается существенно более эффективным, в сравнении со способами скрайбирования, локальных изгибов или плавным волнообразным изменением кристаллографической ориентации поверхности лент. В монокристаллах и поликристаллических лентах стали при их скрайбировании и ЛЛО выявлен резкий рост эффекта снижения магнитных потерь с увеличением степени совершенства кристаллографической текстуры, максимум которого наблюдается в наиболее высоко текстурованных лентах стали. Использование способа ЛЛО и последующей ТМО в переменном магнитном поле позволяет в сталях высших марок с ребровой текстурой, близкой к оптимальной (марка $3409, \mathrm{~B}_{800}=1,93$ Тл), снизить магнитные потери $P_{1,7 / 50}$ на $14-18 \%$ и на $26 \%$ в тонкой аморфной ленте сплава $\mathrm{Fe}_{81} \mathrm{Si}_{7} \mathrm{~B}_{12}$.

\section{Благодарность}

Работа выполнена в рамках государственного задания ФАНО России по теме «Магнит» и при частичной финансовой поддержке Уральского отделения РАН (проект № 15-17-2-53).

\section{Список литературы}

1. Стародубцев Ю. Магнитомягкие материалы : энциклопедический справочник. М. : Техносфера, 2011. -659 c.

2. Ichiyama T. Structure and control of magnetic domain in grain-oriented silicon steel. Journal of the Iron and Steel Institute of Japan. - Vol. 69, iss. 8. - P. 895-902.

3. Сталь электротехническая холоднокатаная анизотропная тонколистовая. ГОСТ 21427.1-83.

4. Пудов В. И., Драгошанский Ю. Н. Перспективность применения аморфнокристаллических покрытий для магнитомягких сплавов // Упрочняющие технологии и покрытия. 2013. - № 8. - С. 44-48.

5. Драгошанский Ю. Н., Пудов В. И. Улучшение магнитной структуры и свойств магнитомягких сплавов при модификации их поверхности. - Физика и химия обработки материалов. - 2013. - № 3. - С. 48-52.

6. Драгошанский Ю. Н., Пудов В. И., Каренина Л. С. Оптимизация доменов и снижение магнитных потерь электротехнической стали активным покрытием и лазерной обработкой. Известия РАН. Серия физическая. - 2013. - Т. 77. - № 10. - С. 1496-1498.

7. Драгошанский Ю. Н., Пудов В. И. Физические основы улучшения электромагнитных свойств магнитомягких материалов / IX Международная научно-техническая конференция «Современные методы и технологии создания и обработки материалов», Минск, 19-22 сентября, 2014 г. - Минск : ФТИ НАН Беларуси, 2014. - № 2. - С. 137-144.

8. Способ изготовления анизотропной электротехнической стали : пат. 2569260 Рос. Федерациия / Пудов В. И., Драгошанский Ю. Н., ИФМ УрО РАН. - 2015, Бюл. № 9. - 3 с.

9. Драгошанский Ю. Н., Пудов В. И. Формирование упорядоченных дефектов и улучшение магнитных характеристик магнитомягких сплавов с различной степенью совершенства текстуры / XI Международная научно-техническая конференция «Современные методы и технологии создания и обработки материалов», Минск, 14-16 сентября, 2016 г. - Минск : ФТИ НАН Беларуси. - 2016. - № 1. - С. 68-73.

10. Драгошанский Ю. Н. Оптимизация доменной структуры и снижение магнитных потерь в анизотропных электротехнических сталях и сплавах / Магнетизм переходных металлов и сплавов : сборник / под ред. Ю. А. Изюмова. - Екатеринбург : УрО РАН, 2000. С. $146-157$.

Dragoshanskii Yu.N. et al. / Prospects for applying laser-mechanical methods to the improvement of the physical properties of transformer steel 
11. Dragoshanskii Yu.N., Pudov V.I. Influence of laser processing and magnetically active inorganic coatings on the dynamic magnetic properties of soft-magnetic materials // Inorganic Materials. - 2013. - Vol. 49, iss. 7. - P. 668-675. - DOI: 10.1134/S0020168513070029 